\title{
IN VIVO STUDY OF FORCES DURING NEEDLE INSERTIONS
}

\author{
B. MAURIN*, L. BARBE*, B. BAYLE*, P. ZANNE*, J.GANGLOFF*, \\ M. DE MATHELIN*, A. GANGI ${ }^{+}$, L. SOLER $^{++}$AND A. FORGIONE ${ }^{++}$ \\ * LSIIT, UMR CNRS-ULP 7005, bd. S. Brant, 67400 Illkirch, France \\ + Département de Radiologie B, Hôpital Civil, 67091 Strasbourg, France \\ ++ IRCAD, Hopital Civil, 67100 Strasbourg, France \\ e-mail: maurin@eavr.u-strasbg.fr
}

\begin{abstract}
Percutaneous procedures are among the developing minimally invasive techniques to treat cancerous diseases of the digestive system. They require a very accurate targeting of the organs, achieved by the combination of tactile sensing and medical imaging. In this paper, we study the forces involved during in vivo percutaneous procedures for the development of a force feedback needle insertion robotic system as well as the development of a realistic simulation device. The paper presents different conditions (manual and robotic insertions) and different organs (liver and kidney). Finally, we review some bio-mechanical models of the literature in the light of our measurements.
\end{abstract}

\section{Introduction}

\subsection{Needle insertion procedures}

Needle insertions are necessary in a wide range of traditional medical treatments like injections or punctures. They are also among the less invasive procedures in developing radiological and surgical techniques like, e.g., the treatment of cancer by radio-frequencies. An increasing number of percutaneous treatments should be expected in the coming years. During these operations, the feedback on the practitioner is both visual and haptic. The visual feedback is given by the position of the inserting point, the needle penetration and usually by intra-operative medical imaging (CT-scan, fluoroscopy, ultrasound). Force feedback is also of central importance. Indeed, this feeback is a great source of information for the practitioner : it may allow him to detect transitions between organs and cavities and even to identify the nature of the organs and the properties of the tissues.

To allow for a precise positioning of the needle in the targeted anatomical structures, these treatments presently require a lengthly procedure with successive image taking and needle insertion steps. As the effectiveness of 
the treatment is strongly dependent on the tip needle position, the practitioner's skill and experience as well as the number of per-operative image taking steps are critical for the outcome of the percutaneous procedure.

For all these reasons a recent interest in robotic devices for needle insertions has arisen ${ }^{9,5}$. Nevertheless, very few projects have yet taken into account the problem of force feedback. This is actually one of the main limitation of pioneer robotic systems. As we believe that the radiologist should remain the principal actor of the operations, such a robotic system should necessarily be tele-operated. Hence real-time force feedback should be necessary. We are presently involved in the design of a force feedback tele-operated robotic system dedicated to CT-guided needle insertions ${ }^{7}$. It will allow, at the same time, a good protection of the practitioner with respect to X-rays, together with visual and haptic feedback. The haptic interface will be also used for simulation and training.

\subsection{Why is it necessary to study forces?}

The nature of the interaction between the needle and the tissues makes the understanding of deformation, cutting and friction mechanisms quite difficult and deserves further detailed examinations. Different studies have been achieved dealing with the measurement and the modeling of forces involved in percutaneous needle insertions ${ }^{3,8}$. They can be classified according to the nature of the bodies in which the needle is inserted: artificial phantoms ${ }^{3}$ or animal tissues ${ }^{8}$. In the first case the authors are specially interested in the correlation between forces and deformations. Such measurements mainly have an interest to build simulators. In the second case, measurements are more interesting from a clinical point of view as they allow to study the behavior of real tissues. Unfortunately, they deal with dead tissues, and in spite of good conditions of preservation ${ }^{8}$, their bio-mechanical properties might be altered. This is specially the case with organs well irrigated by blood, like the liver or the kidney. To our knowledge, very few studies give in vivo measurements of forces in surgical procedures ${ }^{1,2}$ and none of them specifically deals with percutaneous procedures. The main originality of our paper is to characterize the efforts involved in in vivo percutaneous procedures. The experiments detailed in section 2 allow :

i) to establish the ranges of forces involved in percutaneous procedures;

ii) to analyze and model the temporal evolution of the forces during the insertions.

These results are interesting for many reasons. First, they allow to define the design constraints for a robotic system. Then, they help to understand the sensibility of force perception in order to design an haptic interface or a realistic simulator. 


\section{Experimental setup}

We used a Nano-17 6 DOF force sensor from ATI Automation Inc. together with a special handling tool we made to rigidly hold the needle. This device can either be mounted as a robotic arm end-effector or held by hand. Typical resolutions of the sensor are $0.0125 \mathrm{~N}$ along its axis and $0.0625 \mathrm{mN} . \mathrm{m}$ in torque. A dedicated software running on real time Linux OS has been developed allowing synchronized acquisition of measures and robot control.

\subsection{Methods}

All experiments were led with the same 18 gauges $(1.27 \mathrm{~mm}$ diameter), $15.24 \mathrm{~cm}$ biopsy needle with anesthetized pigs. Since the pig is alive, the breathing was artificially stopped for the period of insertion, so that this repetitive perturbation had no impact on the data. Two different methods were used for acquiring in vivo data:

- in the first case a proficient radiologist inserts a needle by manually holding the force device attached to the needle. The task executed by the radiologist was to plunge a needle 4 times into the targeted organs, at a constant velocity, constant depth and with as less shaking as possible. The depth was hard to estimate, approximatively from 30 to $50 \mathrm{~mm}$.

- in the second case the needle insertion is done by a robot, with the assistance of a surgeon. The insertion results of the combination of a descent of $20 \mathrm{~mm}$ in the tissue at constant velocity of $15 \mathrm{~mm} / \mathrm{s}$, then a $8 s$. pause and finally the needle extraction until its initial position at $15 \mathrm{~mm} / \mathrm{s}$.

As we previously underlined, both these experiments serve as an original database, since very few such data exist. Manual measurements allow to characterize the forces that a radiologist is used to feel (see Fig. 1). This is of great interest for engineers as practitioners have difficulties to characterize the forces and torques they apply naturally during operations. Robot insertions also have several benefits (see Fig. 2). In particular, no human intervention is needed except control, hence the same experiment may be repeated several times. The in-

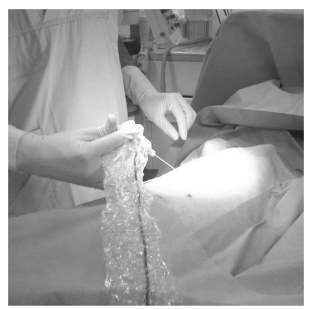

Figure 1. Manual Setup sertions parameters (depth, velocity) can be easily modified, which can help to model the tissues. The main inconvenience in both type of ex- 
periment is that the insertions were carried out "blindly", as we had no imaging device at disposal. Hence no control on the localization and the depth was possible. From ${ }^{8}$, we know that the depth and speed of insertion are of importance. The lack of precise knowledge of the depth of insertion in the organs gave us some difficulties to respect this constraint and we based our insertions on a priori constant depth.

We distinguish the access of organs in two different categories: in the first case the skin is cut superficially (it is a usual practice during percutaneous interventions) and we perforate fasciae, or connective tissues, and some muscles. These experiments are refered to "with skin" in the following. In the second case, all the anatomic layers are cut, thus allowing direct access to the organ. These experiments are refered to "direct access" in the following. Most experiments dealt with the liver but some measurements on kidneys and pancreas were led since they Setup

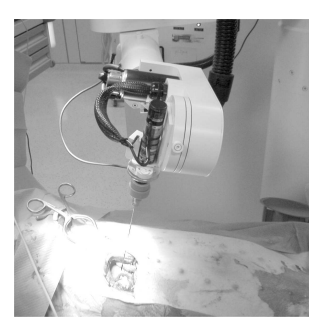

Figure 2. Robotic are also prone to percutaneous procedures.

\subsection{Results}

\subsubsection{Ranges of forces}

We first focused on the maximum exerted forces during insertions. As expected, it appears from statistics, that the most significant results are given by the force along the insertion axis (in other directions, common values are $2 \cdot 10^{-3} \mathrm{~N}$ for forces and $6 \cdot 10^{-3} \mathrm{Nm}$ for torques. As a result, these data are no longer considered in the following analysis.

All the statistics obtained for the radiologist manual insertions are presented in Table 1. The figures for the robotic insertions are presented in

Table 1. Manual insertions

\begin{tabular}{r|cc} 
Organs and method (\# trials) & Maximum force $(N)$ & Std. deviation $(N)$ \\
\hline Liver, with skin (10) & 3.73 & 0.59 \\
Liver removal, with skin (10) & 2.33 & 0.32 \\
Liver, direct access (6) & 0.7 & 0.29 \\
Liver Capsule, direct access (6) & 0.23 & 0.04 \\
Liver removal, direct access (6) & 0.3 & 0.28 \\
Kidney, direct access (5) & 0.74 & 0.54 \\
Pancreas, direct access (5) & 0.83 & 0.28
\end{tabular}

Table 2. Comparing insertion with and without direct access shows that the skin and the muscles accentuate the forces by adding contact forces 
Table 2. Robotic insertions

\begin{tabular}{r|cc} 
Organs and method (\# trials) & Maximum force $(N)$ & Std. deviation $(N)$ \\
\hline Liver, with skin (6) & 1.89 & 0.36 \\
Liver removal, with skin (6) & 0.69 & 0.28 \\
Liver, direct access (6) & 0.59 & 0.17 \\
Liver Capsule, direct access (6) & 0.35 & 0.12 \\
Liver removal, direct access (6) & 0.17 & 0.06 \\
Kidney, direct access (4) & 1.22 & 0.34
\end{tabular}

and elasticity to the global resultant. The differences between manual and robotic insertions is mainly due to the uncontrolled speed and depth of the radiologist.

\subsubsection{Evolution of longitudinal force}

Figure 3 provides a typical plot of an insertion into the liver by direct access. The plot can be split into 3 phases. The descent phase, is the

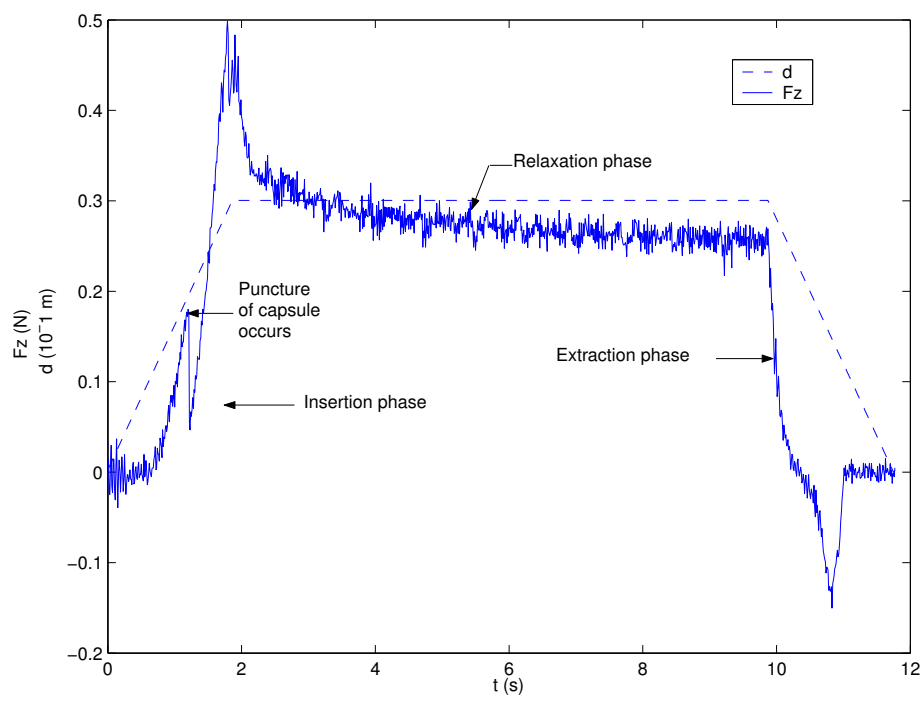

Figure 3. Needle insertions with direct access to the liver

most interesting for force modeling: it corresponds to the succession of an exponential-like rise, a sharp rupture, and finally again a rising slope. It matches the phases felt by the radiologist while piercing the capsule of the organ. The maximum force value is finally reached when the movement 
ceases. As a consequence of organ elasticity, this peak is rather proportional to the depth of penetration. The second phase corresponds to the relaxation of the tissues. Not surprisingly, even if the needle is motionless, we have a slow decreasing force applied along the axis of the needle. This is known as a repulsion force applied on the tip. It depends mainly on the kind of bevel the needle is made of. For our experiment a regular bevel was used and the liver relaxation converge to a constant repulsion force of $0.3 \mathrm{~N}$ in average. Finally, the last phase corresponds to the removal of the needle during which only friction forces are applied on the surface of the needle: after a fall to $0 N$, we obtain an exponential-like plot as in the insertion phase.

Figure 4 is a plot allowing the comparison between the manual and the robotic insertions in the case of the liver "with skin".

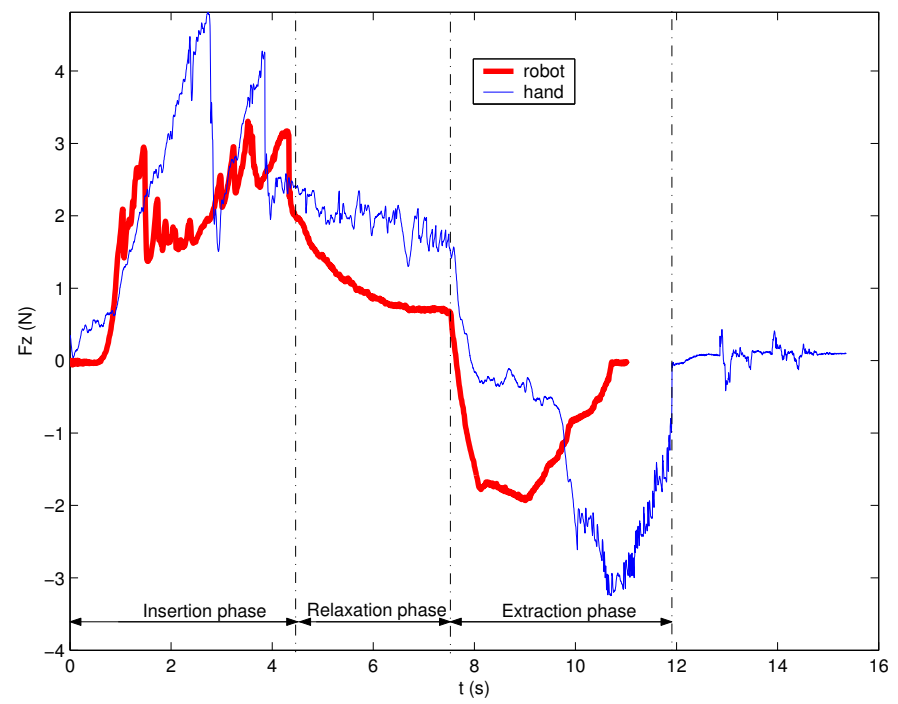

Figure 4. Radiologist and a robotic insertions with skin access to the liver.

\subsection{Modeling of the Liver}

To model the insertion into the liver, we compared two models fitted with our measurements. Simone and Okamura ${ }^{8}$ model the forces by the sum of 3 components: stiffness, friction and cutting. We will only stress the stiffness force and friction force as the test procedure was not the same as the authors 
did. The stiffness force is exemplified during the insertion phase, before the puncture in the capsule. A second order polynomial $f(d)=a_{0}+a_{1} d+a_{2} d^{2}$ that relates depth to force, is well suited for this curve. After data fitting, the following coefficients were obtained: $a_{0}=0 \mathrm{~N}, a_{1}=0.002 \mathrm{~N} / \mathrm{mm}$ and $a_{2}=0.0023 \mathrm{~N} / \mathrm{mm}^{2}$. These values are very close to those exposed in ${ }^{8}$. The friction force is given by a Karnopp ${ }^{8}$ model and we found a positive static friction of $0.121 \mathrm{mN} / \mathrm{mm}$ and a negative of $-0.117 \mathrm{mN} / \mathrm{mm}$. The dynamic friction coefficients were considered null (force proportional to distance). The positive damping coefficient was $3.2 \mathrm{mNs} / \mathrm{mm}^{2}$ where the negative was $1.6 \mathrm{mNs} / \mathrm{mm}^{2}$.

The second model we tested is taken from Maurel ${ }^{6}$ and based on the work of Fung ${ }^{4}$. The insertion has two phasis: before the capsule puncture and after. In each phase, the force is modeled as an exponential function of the depth : $f(d)=\left(F_{0}+b\right) e^{a\left(d-d_{0}\right)}+b$. With a NewtonRaphson optimization we found the parameter vector $\theta=\left[a, b, d_{0}, F_{0}\right]$ before the puncture: $\theta=[0.121,-0.098,11.45,0.2]$; and after the puncture: $\theta=[-0.031,1.7,19.61,-3.39]$.

In Fig. 5, the two models are drawn for comparison. For the first model we get a mean error $\bar{f}=7.4 \mathrm{mN}$ and a standard deviation $\sigma=0.69 \mathrm{mN}^{2}$; for the second $\bar{f}=0.79 \mathrm{mN}$, and $\sigma=0.31 \mathrm{mN}^{2}$. As expected, the errors are low and the models are both of interest.
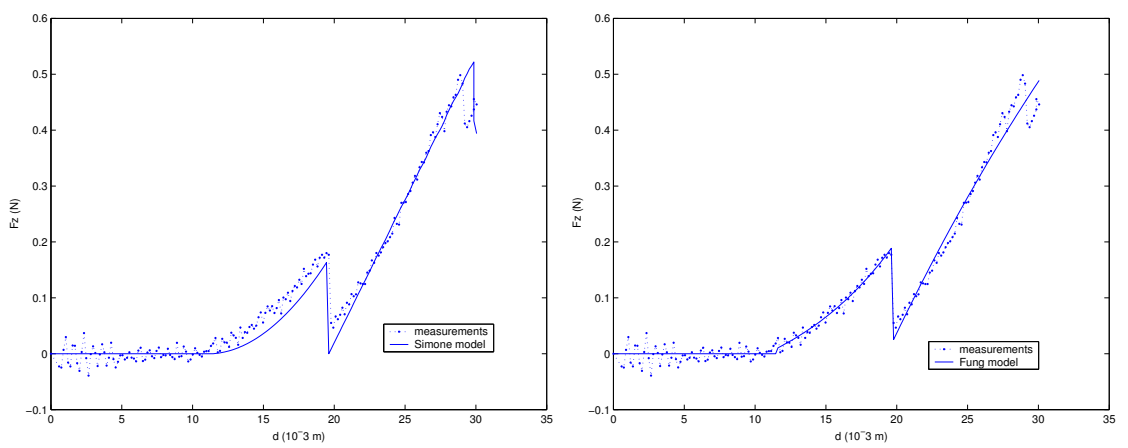

Figure 5. Two models fitted with our data (left: Simone, right: Maurel)

\section{Conclusion}

This paper presents data analysis to characterize the values of forces during in vivo needle insertions. It underlines both the necessary sensibility to render the organs transitions and the important forces required to pierce 
the tissues. As an application, models are derived from those data.

Futur work will include the measure of the depth insertion and eventually of the organs deformations. Another point to develop would be to get data during a real human intervention. This was prepared in our experimental setup as the mechanical parts of the sensor have the particularity to possibly be protected by a sterilized bag

\section{Acknowledgments}

The authors wish to thank the Region Alsace Council and the CNRS ROBEA program for their financial contribution to this project.

\section{References}

1. I. Brouwer, J. Ustin, L. Bentley, A. Sherman, N. Dhruv, and F. Tendick. Measuring in vivo animal soft tissue properties for haptic modeling in surgical simulation. In Medicine Meets Virtual Reality 2001, pages 69-74, 2001.

2. J. D. Brown, J. Rosen, Y. S. Kim, L. Chang, M. N. Sinanan, and B.Hannaford. In-vivo and in-situ compressive properties of porcine abdominal soft tissues. In Medicine Meets Virtual Reality 2001, pages 26-32, January 2003.

3. S. P. DiMaio and S. E. Salcudean. Needle insertion modelling and simulation. In Proceedings of the 2002 IEEE International Conference on Robotics and Automation, pages 2098-2105, Washington DC, USA, May 2002. ICRA'02.

4. Y. C. Fung. Biomechanics: mechanical properties of living tissues. SpringerVerlag, second edition edition, 1993.

5. J. Hong, T. Dohi, M. Hashizume, K. Konishi, and N. Hata. An ultrasounddriven needle-insertion robot for percutaneous cholecystostomy. Physics in Medicine and Biology, pages 441-455, 2004. IOP Publishing Ltd.

6. W. Maurel. 3D Modeling of the Human Upper Limb Including the Biomechanics of Joints, Muscles and Soft Tissues. PhD thesis, Laboratoire d'Infographie - Ecole Polytechnique Federale de Lausanne, 1999.

7. B. Maurin, O. Piccin, B. Bayle, J. Gangloff, M. de Mathelin, P. Zanne, C. Doignon, A. Gangi, and L. Soler. A new robotic system for ct-guided percutaneous procedures with haptic feedback. In Proceedings of the $2004 \mathrm{Com}$ puter Assisted Radiology and Surgery Congress (to appear), Chicago, USA, June 2004. CARS'04.

8. C. Simone and A. M. Okamura. Modeling of needle insertion forces for robotassisted percutaneous therapy. In Proceedings of the 2002 IEEE International Conference on Robotics and Automation, pages 2085-2091, Washington, DC, USA, May 2002. ICRA'02.

9. D. Stoianovici, L. L. Whitcomb, J. H. Anderson, R. H. Taylor, and L. R. Kavoussi. A modular surgical robotic system for image guided percutaneous procedures. In Proceedings of the 1998 International Conference on Medical Image Computing and Computer-Assisted Intervention, Cambridge, MA, October 1998. MICCAI'98. 